Thermorheological Properties Near the Glass Transition of Oligomeric Poly(methyl methacrylate) Blended with Acrylic Polyhedral Oligomeric Silsesquioxane Nanocages

Edward T. Kopesky, Stephen G. Boyes, Neil Treat, Robert E. Cohen, Gareth H. McKinley

August 26, 2005 HML Report Number 05-P-13 


\title{
Thermorheological Properties Near the Glass Transition of Oligomeric Poly(methyl methacrylate) Blended with Acrylic Polyhedral Oligomeric Silsesquioxane Nanocages
}

Edward T. Kopesky ${ }^{1}$, Stephen G. Boyes ${ }^{2}$, Neil Treat ${ }^{3}$, Robert E. Cohen ${ }^{1}$, Gareth H. McKinley $^{4} *$

${ }^{1}$ Department of Chemical Engineering, Massachusetts Institute of Technology, ${ }^{2}$ Department of Chemistry and Geochemistry, Colorado School of Mines, ${ }^{3}$ School of Polymers and High Performance Materials, University of Southern Mississippi, ${ }^{4}$ Department of Mechanical Engineering, Massachusetts Institute of Technology

*Email: gareth@mit.edu

Telephone: (617) 258-0754

Fax: (617) 258-8559

August 26, 2005

\begin{abstract}
Two distinct oligomeric species of similar mass and chemical functionality $\left(M_{\mathrm{w}} \approx 2,000 \mathrm{~g} / \mathrm{mol}\right.$ ), one a linear methyl methacrylate oligomer (radius of gyration $R_{\mathrm{g}} \approx$ $1.1 \mathrm{~nm}$ ) and the other a hybrid organic-inorganic polyhedral silsesquioxane nanocage (methacryl-POSS, $r \approx 1.0 \mathrm{~nm}$ ), were subjected to thermal and rheological tests to compare the behaviors of these geometrically dissimilar molecules over the entire composition range. The glass transition temperatures of the blends varied monotonically between the glass transition temperatures of the pure oligomer $\left(T_{\mathrm{g}}=-47.3^{\circ} \mathrm{C}\right)$ and the pure POSS $\left(T_{\mathrm{g}}=-61.0^{\circ} \mathrm{C}\right)$. Blends containing high POSS contents (with volume fraction $\left.\phi_{\text {POSS }} \geq 0.90\right)$ exhibited enhanced enthalpy relaxation in DSC measurements, and the degree of enthalpy relaxation was used to calculate the kinetic fragility indices $m$ of the oligomeric MMA $(m=59)$ and the POSS $(m=74)$. The temperature dependences of the viscosities were fitted by the free volume-based WLF-VFT framework and a dynamic scaling relation. The calculated values of the fragility from the WLF-VFT fits were similar for the POSS $(m=82)$ and for the oligomer $(m=76)$, and the dynamic scaling exponent was similar for the oligomeric MMA and the POSS. Within the range of known fragilities for glass-forming liquids, the temperature dependence of the viscosity was found to be similarly fragile for the two species. The difference in shape of the nanocages and oligomer chains is unimportant in controlling the glass-forming properties of the blends at low volume fractions $\left(\phi_{\mathrm{POSS}}<0.20\right)$; however, at higher volume fractions, adjacent POSS cages begin to crowd each other, leading to an increase in the fractional free volume at the glass transition temperature and the observed enhanced enthalpy relaxation in DSC.
\end{abstract}




\section{Introduction}

\subsection{Previous work}

In recent studies of the thermorheological properties of polymer nanocomposites, unusual properties have been observed when the nanoparticle size approaches the molecular scale $(d<10 \mathrm{~nm})$. Such molecular composites exhibit widely differing properties depending on the particle-matrix interaction (Zhang and Archer 2002; Mackay et al. 2003). Mackay et al. examined an athermal polymeric molecular composite system consisting of entangled polystyrene(PS) melts $\left(7.5 \mathrm{~nm} \leq R_{\mathrm{g}} \leq 15 \mathrm{~nm}\right)$ filled with highly crosslinked polystyrene particles $(3 \mathrm{~nm} \leq r \leq 5 \mathrm{~nm})$ and found significant plasticization of the linear PS matrix by the PS nanoparticles. A recent study by Capaldi et al. (2005) reported atomistic simulations of an oligomeric molecular composite system of oligomeric polyethylene $\left(\mathrm{PE}, \mathrm{C}_{50} \mathrm{H}_{102}\right)$ containing polyhedral oligomeric silsesquioxanes (cyclopentyl-POSS $\left[\left(\mathrm{C}_{5} \mathrm{H}_{9}\right)_{8} \mathrm{Si}_{8} \mathrm{O}_{12}\right]$ ) at POSS loadings of 5, 15, and $25 \mathrm{wt} \%$. The addition of cyclopentyl-POSS to the matrix caused a $22 \%$ decrease in the diffusion coefficient of the PE at a POSS loading of $25 \mathrm{wt} \%$ at $T=500 \mathrm{~K}$. However, continuous aggregation of the POSS nanocages was also observed on the time scale of the simulations ( $\Delta t=10 \mathrm{~ns})$; thus it remains unclear whether a well-dispersed oligomeric molecular composite system would exhibit similar properties.

Polyhedral oligomeric silsesquioxanes possess the hybrid organic-inorganic structure $R_{x} T_{x}$, where $T$ represents the silsesquioxane linkage $\mathrm{SiO}_{3 / 2}$, R represents an organic group, and the subscript $\mathrm{x}$ denotes the number of silsesquioxane linkages in the cage. In Figure 1 we show a $\mathrm{T}_{10}$ POSS nanocage with acrylic R-groups, which has a 
characteristic size $r \approx 1.0 \mathrm{~nm}$ as measured from the center of the cage to the outer atom of one of the R-groups.

In our initial study of POSS-poly(methyl methacrylate)(PMMA) blends (Kopesky et al. 2004), we incorporated two monodisperse, crystallizable $\mathrm{T}_{8}$ POSS components into PMMA: cyclohexyl-POSS and isobutyl-POSS. Each was blended separately with PMMA and we observed a strong thermodynamic driving force toward phase separation of the POSS into crystallites with observed diameters in the range $50 \mathrm{~nm} \leq d \leq 5 \mu \mathrm{m}$. There was evidence of a small amount of molecularly-dispersed POSS in each case, indicated by a slight $(\approx 10 \%)$ decrease in the zero shear-rate viscosity at low volume fractions of $\phi_{\text {POSS }} \leq 0.03$. However, the concurrent crystallization of POSS masked the effect of welldispersed POSS on the time-temperature shift factors, which we examined within the Williams-Landel-Ferry (WLF) framework (Ferry 1980):

$$
\log a_{T}=\frac{-c_{1}\left(T-T_{r}\right)}{c_{2}+\left(T-T_{r}\right)}
$$

where $c_{1}$ and $c_{2}$ are constants, and $T_{\mathrm{r}}$ is the reference temperature. The use of a POSScontaining copolymer as the matrix material improved dispersion of the POSS filler, but extensive crystallite formation was still observed above $\phi \approx 0.10$. To avoid crystallization issues, we performed a subsequent study that examined the thermorheological effects of a polydisperse, noncrystallizable POSS species that contained acrylic R-groups (methacrylPOSS) (Kopesky et al. 2005). We found that methacryl-POSS could disperse in PMMA up to a loading of $\phi_{\mathrm{POSS}} \approx 0.10$ before phase separation became significant. The glass transition temperature $T_{\mathrm{g}}$ decreased by approximately $10^{\circ} \mathrm{C}$ up to $\phi_{\mathrm{POSS}}=0.10$ before plateauing due to progressive phase separation. A monotonic increase in the fractional 
free volume as determined from the time-temperature shift factors was observed at loadings $\phi \leq 0.20$, consistent with the decrease in the glass transition temperature values over this range of POSS content.

In the present study we examine a system that is similar to the methacrylPOSS-PMMA blends described above; however we select an oligomeric MMA $\left(M_{\mathrm{w}}=2160 \mathrm{~g} / \mathrm{mol}\right.$, radius of gyration $\left.R_{\mathrm{g}} \approx 1.1 \mathrm{~nm}\right)$ as the polymeric component rather than a high molecular weight PMMA matrix in order to obtain complete miscibility of the two components over the entire composition range. In addition, the oligomer and the methacryl-POSS $\left(M_{\mathrm{w}}=2025 \mathrm{~g} / \mathrm{mol}\right)$ have similar molecular weights but vastly different shapes. The present system is similar to the oligomeric molecular composites simulated by Capaldi et al. (2005) but the polydispersity of the methacryl-POSS nanocage sample completely eliminates crystallization. As before, we examine our results using the WLF framework in order to understand better the molecular-level mechanisms responsible for the observed rheological behavior. In addition, we utilize a dynamic scaling model to add support to our conclusions.

\subsection{Theory}

The effect of a diluent on the viscosity of a polymeric material is important commercially and, in particular, the temperature dependence of the viscosity in the region $T_{\mathrm{g}} \leq T \leq T_{\mathrm{g}}+100^{\circ} \mathrm{C}$ has drawn considerable attention because of the complex relaxation processes near $T_{\mathrm{g}}$ (Ferry 1980). The familiar Arrhenian dependence of the viscosity on temperature is valid for temperatures $T>T_{\mathrm{g}}+100^{\circ} \mathrm{C}$ and is given by:

$$
\eta_{0}(T)=\eta_{0}\left(T_{r}\right) \exp \left[\frac{\Delta H}{R}\left(\frac{1}{T}-\frac{1}{T_{r}}\right)\right]
$$


where $\eta_{0}$ is the zero shear-rate viscosity, $\Delta H$ is an activation energy for flow, and $R$ is the universal gas constant. This relationship breaks down as $T_{\mathrm{g}}$ is approached from above, causing the value of $\Delta H$ to increase with decreasing temperature. The deviation from Arrhenian behavior is often quantified using a parameter known as the kinetic fragility $m$ (Angell 1991; Angell 1995), which can be defined as (Plazek and Ngai 1991; Bohmer and Angell 1992):

$$
m=\left(\frac{\partial \log \eta_{0}}{\partial\left(T_{g} / T\right)}\right)_{T_{g}}
$$

The fragility is often called the "steepness index" (Plazek and Ngai 1991; Wang et al. 2002), as it denotes how abruptly the viscosity increases at the glass transition temperature.

A variety of semi-empirical models have been developed to describe the deviation of a liquid from Arrhenian behavior. Perhaps the most well-known such model is the Vogel-Fulcher-Tammann (VFT) equation (Vogel 1921; Fulcher 1925; Tammann and Hesse 1926):

$$
\eta_{0}=A \exp \left[\frac{B}{\left(T-T_{0}\right)}\right]
$$

where $A$ and $B$ are constants and $T_{0}$ is the familiar Vogel temperature (often written as $T_{\infty}$ ) which represents the temperature at which the viscosity diverges. In general, experiments indicate that $T_{0} \approx T_{\mathrm{g}}-50 \mathrm{~K}$ (Ferry 1980). Equation 4 may also be written as:

$$
\eta_{0}=A \exp \left[\frac{D T_{0}}{\left(T-T_{0}\right)}\right]
$$


where the dimensionless constant $D=B / T_{0}$ is an inverse measure of the fragility of the liquid (Angell 1995).

The VFT equation is identical in form to the WLF equation [equation (1)] when two substitutions are made (Ferry 1980):

$$
\begin{aligned}
& c_{1}=\frac{B}{2.303\left(T_{r}-T_{0}\right)} \\
& c_{2}=T_{r}-T_{0}
\end{aligned}
$$

A separate model proposed recently by Colby (2000) was developed by visualizing a liquid near the glass transition as a set of cooperatively rearranging domains (Adam and Gibbs 1965). These domains increase in size as the temperature is lowered, and at a critical temperature $T_{\mathrm{c}}$, the size of these domains diverges, leading to percolation and an infinite viscosity. The model has the form (Stanley 1971; Hohenberg and Halperin 1977):

$$
\eta_{0} \propto\left[\frac{\left(T-T_{c}\right)}{T_{c}}\right]^{-v z}
$$

where $z$ is the dynamic exponent and the exponent $v$ controls the divergence in size of the cooperatively rearranging domains. Values of the critical temperature $T_{\mathrm{c}}$ are usually significantly higher than the Vogel temperature $T_{0}$ obtained from equation (4) (and in the range $\left.T_{\mathrm{c}} \approx T_{0}+40 \mathrm{~K}\right)$ (Colby 2000), and thus $T_{\mathrm{c}}$ is much closer to the glass transition temperature than $T_{0}$. For polymers, the product of the exponents $v z$ in equation (8) typically falls within the range $8 \leq v z \leq 11$ (Colby 2000).

In the present study we utilize both the WLF-VFT formalism [equations (1), (4)(7)] and the dynamic scaling relations of Colby [equation (8)]. We use these models to 
help elucidate the underlying mechanisms for the observed viscosity-temperature behavior of our molecular composite blends of oligomeric-MMA and methacryl-POSS nanocages.

\section{Experimental Section}

\subsection{Oligomer Synthesis}

The catalytic chain transfer agent bis(boron difluorodimethylgloximate) cobaltate(II) (COBF) was synthesized according to a modification of the method described by Bakac and Espenson (Bakac and Espenson 1984; Bakac et al. 1986; Sanayei and O'Driscoll 1989; Suddaby et al. 1997). Methyl methacrylate (MMA) (Fisher) was purified by passing through a column of activated basic alumina. Toluene and MMA were deoxygenated by purging with nitrogen for $1 \mathrm{~h}$ before use. The initiator 2,2'Azoisobutyronitrile (AIBN) (Aldrich) was purified by recrystallization from methanol.

The reaction was performed using standard Schlenk apparatus under oxygen free conditions (Shriver and Drezdzon 1986). Initially the MMA and solvent solution (100 mL MMA, $50 \mathrm{~mL}$ Toluene) was purged using nitrogen for at least 1 hour. The AIBN (100 mg) and COBF (6 mg) were added to a separate flask, with a magnetic stirrer bar, sealed with a septum, and deoxygenated by repeated vacuum/nitrogen back-filling cycles. The deoxygenated MMA/toluene mixture was then transferred to the flask containing the AIBN and COBF via a cannular, and the flask was heated at $70{ }^{\circ} \mathrm{C}$ for 24 hours under an atmosphere of nitrogen. After this time, the reaction solution was passed through a column of activated basic alumina to remove any residual catalyst and then the residual monomer and solvent were removed by heating at $60{ }^{\circ} \mathrm{C}$ under vacuum for 24 hours. 
The molecular weight distribution was determined by size exclusion chromatography (SEC) consisting of a Waters Alliance 2659 Separations Module, an online multiangle laser light scattering (MALLS) detector (MiniDAWN ${ }^{\mathrm{TM}}$, Wyatt Technology Inc.), an interferometric refractometer (Optilab DSP ${ }^{\mathrm{TM}}$, Wyatt Technology Inc.) and two Plgel $3 \mu \mathrm{m}$ Mixed-E columns in series. The eluent was tetrahydrofuran (THF) kept at $35^{\circ} \mathrm{C}$ with a rate of $1.000 \mathrm{~mL} / \mathrm{min}$. Sample concentrations were $5 \mathrm{mg} / \mathrm{mL}$ in freshly distilled THF, and the injection volume was $100 \mu \mathrm{L}$. The weight-average molecular weight was determined to be $M_{\mathrm{w}}=2190 \mathrm{~g} / \mathrm{mol}$ and the polydispersity index $M_{w} / M_{n}=1.56$.

\subsection{Other Materials}

The methacryl-POSS sample was purchased from Hybrid Plastics (Hattiesburg, MS) and used as received. ${ }^{29} \mathrm{Si}$ NMR showed that the methacryl-POSS was a cage mixture composed of $T_{8}, T_{10}, T_{12}$, and $T_{14}$ cages, with $T_{10}$ cages accounting for nearly half the mixture ( $\approx 47 \mathrm{wt} \%$ ) (Kopesky et al. 2005).

\subsection{Blending}

Both the oligomeric MMA and the methacryl-POSS are liquids at room temperature. The oligomer has approximately the consistency of honey while the methacryl-POSS is noticeably less viscous, more akin to motor oil. These species were blended together by weighing out the oligomer portion first in a glass vial and subsequently adding the methacryl-POSS using a micro spatula. Moderate heating was required $\left(T=50^{\circ} \mathrm{C}\right.$ for 5 minutes) in order to homogenize the viscous mixtures. Compositions ranged from pure oligomer $\left(\phi_{\mathrm{POSS}}=0.00\right)$ to pure POSS $\left(\phi_{\mathrm{POSS}}=1.00\right)$. 


\subsection{Differential Scanning Calorimetry}

Differential scanning calorimetry (DSC) was performed on a TA Instruments Q1000 using hermetically-sealed sample pans. Samples were cooled from $T=50^{\circ} \mathrm{C}$ to $T=-90^{\circ} \mathrm{C}$ at a cooling rate of $q=3^{\circ} \mathrm{C} / \mathrm{min}$, held for five minutes, then heated at $3{ }^{\circ} \mathrm{C} / \mathrm{min}$ to $T=50^{\circ} \mathrm{C}$. The glass transition temperature of the blends was taken as the inflection point in the heat flow versus temperature curve. In the tests to determine the limiting fictive temperature $T_{\mathrm{f}}^{\prime}$ [see equation (11) below], the cooling rate was varied over the range $0.2^{\circ} \mathrm{C} / \mathrm{min} \leq q \leq 30^{\circ} \mathrm{C} / \mathrm{min}$ and the heating rate was kept constant at $10^{\circ} \mathrm{C} / \mathrm{min}$.

\subsection{Rheology}

Rheological measurements over the temperature range $0^{\circ} \mathrm{C} \leq T \leq 40^{\circ} \mathrm{C}$ were performed on a TA Instruments AR2000 rheometer using a cone-and-plate geometry (20 $\mathrm{mm}$ cone diameter, $2^{\circ}$ cone angle, $58 \mu \mathrm{m}$ truncation height). The temperature was controlled by a Peltier plate system. Linear viscoelastic tests were performed at $T=0^{\circ} \mathrm{C}$ at a strain amplitude $\gamma^{\circ}=0.05$. Additional low temperature viscometric tests were performed using the Environmental Test Chamber (ETC) with liquid nitrogen as the coolant over the temperature range $-55^{\circ} \mathrm{C} \leq T \leq 0^{\circ} \mathrm{C}$.

\section{Results}

\subsection{Differential Scanning Calorimetry}

Differential scanning calorimetry (DSC) scans of the methacryl-POSS-oligomer blends are reproduced in Figure 2. Each heat flow-temperature curve shows only one glass transition, indicating complete miscibility over the entire composition range. The values of the glass transition temperature $T_{\mathrm{g}}$ (Figure 3 ) decrease in a concave-upward 
fashion with increasing POSS content. From Figure 2 it is also apparent that the glass transition region is sharper at higher POSS contents. Additionally, the blends containing $\phi_{\mathrm{POSS}} \geq 0.90$ show a local minimum of increasing depth just beyond the glass transition $\left(T \approx-58^{\circ} \mathrm{C}\right)$. This peak is characteristic of enthalpy relaxation below $T_{\mathrm{g}}$ as the nonequilibrium glass slowly approaches equilibrium (Struick 1978).

The monotonic decrease in the glass transition temperatures of the blends with increasing POSS content agrees qualitatively with the prediction of the Kelley-Bueche equation (Kelley and Bueche 1961):

$$
T_{g}=\frac{\left\lfloor\phi_{1} \alpha_{f 1} T_{g 1}+\left(1-\phi_{1}\right) \alpha_{f 2} T_{g 2}\right\rfloor}{\phi_{1} \alpha_{f 1}+\left(1-\phi_{1}\right) \alpha_{f 2}}
$$

where $\alpha_{f 1}$ and $\alpha_{f 2}$ are the respective thermal expansion coefficients of the free volume, $T_{\mathrm{g} 1}$ and $T_{\mathrm{g} 2}$ are the respective glass transition temperatures, and $\phi_{1}$ is the volume fraction of component 1 . The decrease in $T_{\mathrm{g}}$ with increasing POSS content also highlights a difference between our completely miscible system and the simulated cyclopentylPOSS-polyethylene system of Capaldi et al. (2005). The slowdown in diffusion observed in the simulations of Capaldi et al. would be expected to translate into an increase in glass transition temperature with increasing POSS content, the opposite of what our POSSoligomer system shows. The absence of a driving force for crystallization in our system shows unambiguously that the methacryl-POSS has a plasticizing effect on the oligomeric MMA for all compositions analyzed. 
Additional DSC measurements were performed in order to determine the effect of cooling rate $q$ on the limiting fictive temperature $T_{f}^{\prime}$. The relation between these two variables can be modeled as an activated rate process (Moynihan et al. 1976):

$$
-\frac{\partial \ln q}{\partial\left(1 / T_{f}^{\prime}\right)}=\frac{\Delta H^{*}}{R}
$$

where $\Delta H^{*}$ is the Arrhenian activation energy from equation (2) at $T=T_{\mathrm{g}}$ and $T_{f}^{\prime}$ is defined by the expression (Moynihan et al. 1976):

$$
\int_{T_{h i}}^{T_{f}{ }^{\prime}}\left(C_{p l}-C_{p g}\right) d T_{f}=\int_{T_{h i}}^{T_{l o}}\left(C_{p}-C_{p g}\right) d T
$$

In the above expression, $T_{l o}$ is a temperature sufficiently below the glass transition region that the heat capacity of the material is the same as its glassy value $C_{p g}$, and $T_{h i}$ is a temperature sufficiently far above the glass transition region that the heat capacity has reached its liquid value $C_{p l}$. The inset to Figure 4 is a graphical depiction of how the limiting fictive temperature $T_{f}^{\prime}$ is determined. The data in Figure 4 consist of the heat capacity $C_{\mathrm{p}}$ plotted against temperature for both the methacryl-POSS and the MMA oligomer at cooling rates of $0.2 \leq q \leq 10^{\circ} \mathrm{C} / \mathrm{min}$. Both samples show significant enthalpy relaxation at the slowest cooling rate of $0.2^{\circ} \mathrm{C} / \mathrm{min}$, however the peak in $C_{\mathrm{p}}$ is much taller and sharper in the POSS spectrum. The heat capacity curve for the POSS passes through a maximum at all cooling rates investigated while in the oligomer such a peak is only clearly observed at the two slowest cooling rates.

By utilizing equation (10), we can determine the activation energy for structural relaxation $\Delta H^{*}$ by plotting the natural logarithm of the cooling rate $q$ against the reciprocal of the limiting fictive temperature $T_{f}^{\prime}$, as has been done in Figure 5. Linear 
regression of the data for the POSS and also for the oligomer produced high correlation coefficients of $R^{2} \geq 0.99$, indicating that equation (10) describes the data well over this range of cooling rates. The resultant value of $\Delta H^{*}$ for the POSS $\left(\Delta H^{*}=302 \mathrm{~kJ} / \mathrm{mol}\right)$ is $19 \%$ greater than that for the oligomer $\left(\Delta H^{*}=254 \mathrm{~kJ} / \mathrm{mol}\right)$. This activation energy for structural relaxation is directly proportional to the fragility $m$ of a glass-forming liquid [equation (3)] according to the expression (Plazek and Ngai 1991; Wang et al. 2002):

$$
m=\frac{\Delta H^{*}}{2.303 R T_{g}}
$$

Interestingly, the methacryl-POSS has a higher fragility index $(m=74)$ than the oligomer $(m=59)$ as determined from DSC experiments; however, the difference in fragilities is small when compared with the range of known $m$ values for glass-forming liquids (20 $\leq m \leq 191)$ (Angell 1995; Ding et al. 2004). This result will be revisited below when we quantify the fragility independently using viscometric tests.

\subsection{Linear Viscoelastic Properties}

The linear viscoelastic properties of the methacryl-POSS-oligomer blends were measured in small amplitude oscillatory shear flow and the storage moduli $G^{\prime}$ and the loss moduli $G^{\prime \prime}$ are plotted in Figure 6 at a reference temperature $T_{0}=0^{\circ} \mathrm{C}$. The pure oligomer has a measurable amount of elasticity $\left(G^{\prime} \approx 10^{4} \mathrm{~Pa}\right.$ at $\left.\omega=10^{2} \mathrm{rad} / \mathrm{s}\right)$ and the storage modulus data show the expected slope of 2 observed in simple viscoelastic fluids. A one mode Maxwell fit to the data for the oligomer produced the parameters $\eta_{1}=3180 \mathrm{~Pa}$ s and $\lambda_{1}=5.1 \times 10^{-4} \mathrm{~s}$. The blends with small loadings of POSS ( $\phi_{\mathrm{POSS}} \leq$ 0.10 ) have monotonically decreasing elasticity but retain nearly the same linear slope of 2 on the log-log scale. The pure POSS exhibits a more complex shape in $G^{\prime}$. A slope of 
approximately 1.8 is observed at a reduced frequency $a_{\mathrm{T}} \omega>200 \mathrm{rad} / \mathrm{s}$, however an apparent shoulder appears at $\omega<200 \mathrm{rad} / \mathrm{s}$ over which the data exhibit a shallower slope. Eventually, at very low reduced frequencies $a_{\mathrm{T}} \omega \leq 1 \mathrm{rad} / \mathrm{s}$, the POSS appears to enter a terminal relaxation regime. Evidence of this shoulder in $G^{\prime}$ is also visible in the blends with POSS contents $\phi_{\mathrm{POSS}} \geq 0.20$. The onset of this intermediate relaxation regime shifts to higher frequencies and lower values of $G^{\prime}$ as the POSS content increases.

\subsection{Viscometric Properties}

The zero shear-rate viscosity at $T=20^{\circ} \mathrm{C}$ is plotted against the volume fraction of methacryl-POSS in Figure 7. The values of $\eta_{0}$ for the binary mixtures of POSS and oligomer fall well below the prediction of the simple log viscosity expression expected for mixtures of polymeric liquids (Bird et al. 1987):

$$
\log \eta_{0}=\phi_{\text {POSS }} \log \eta_{\text {POSS }}+\left(1-\phi_{\text {POSS }}\right) \log \eta_{\text {oligo }}
$$

where $\eta_{\text {POSS }}$ and $\eta_{\text {oligo }}$ are the zero shear-rate viscosities of the POSS and the oligomer, respectively.

In Figure 8(i), we plot the variation in the zero shear-rate viscosity $\eta_{0}$ with the reciprocal of the absolute temperature. Shift factors $a_{\mathrm{T}}\left(T, T_{\mathrm{r}}\right)$ were obtained from the temperature dependence of the viscosity well above $T_{\mathrm{g}}$, over the range $0^{\circ} \mathrm{C} \leq T \leq 40^{\circ} \mathrm{C}$. These shift factors were then fit to the Arrhenius model defined by equation (2):

$$
\log a_{T}=\frac{\Delta H}{2.303 R}\left(\frac{1}{T}-\frac{1}{T_{r}}\right)
$$

Regression fits to all sample sets yielded approximately the same correlation coefficients $\left(R^{2} \approx 0.998\right)$ for plots of $\log a_{\mathrm{T}}$ vs. $1 / \mathrm{T}$. Values of the flow activation energy $\Delta H$ are plotted in Figure 8(ii). The dotted lines in Figure 8(i) represent Arrhenian fits to the 
POSS and the oligomer data sets over the range $0 \leq T \leq 40^{\circ} \mathrm{C}$. As expected, it is apparent from both fits that the data taken at $T<0^{\circ} \mathrm{C}$ diverge from the calculated Arrhenian fits to the viscosity; thus a thermorheological model with an additional material parameter is required to account for rapid increase in magnitude of the flow activation energy $\Delta H$ near $T_{\mathrm{g}}$

\section{Discussion}

We now report the results from our attempts to fit the viscosity data over the entire temperature range investigated. Our analysis begins with the WLF equation [equation (1)]. The quantity $-\left(T-T_{r}\right) / \log a_{T}$ is plotted against $\left(T-T_{r}\right)$ in Figure 9(i) at a reference temperature $T_{\mathrm{r}}=20^{\circ} \mathrm{C}$ (Ferry 1980). The WLF constants $c_{1}$ and $c_{2}$ were calculated from the slope $a$ and the y-intercept $b$ of linear regression fits to the data in Figure 9(i):

$$
\begin{aligned}
& c_{1}=1 / a \\
& c_{2}=b / a
\end{aligned}
$$

Overall, the data are linear on both sides of the reference temperature $T_{\mathrm{r}}=20^{\circ} \mathrm{C}$, with a moderate amount of noise centered on the best-fit line. The values of $c_{1}$ and $c_{2}$ are reported in Table 1 . The constants $c_{1}^{g}$ and $c_{2}{ }^{g}$, which are the WLF coefficients at the glass transition temperature, may be calculated using the relations (Ferry 1980):

$$
\begin{aligned}
& c_{1}^{g}=\frac{c_{1} c_{2}}{\left(c_{2}+T_{g}-T_{r}\right)} \\
& c_{2}^{g}=c_{2}+T_{g}-T_{r}
\end{aligned}
$$


The constant $c_{1}^{g}$ was used to approximate the fractional free volume $f_{\mathrm{g}}$ at the glass transition temperature (Ferry 1980):

$$
f_{g}=\frac{B_{W L F}}{2.303 c_{1}^{g}}
$$

where $B_{W L F}$ is a constant usually assumed to be unity, and these values are also included in Table 1. For comparison, our previous study of high molecular weight PMMA yielded a value for the free volume at $T_{\mathrm{g}}$ of $f_{\mathrm{g}} / \mathrm{B}=0.030 \pm 0.001$ (Kopesky et al. 2005). The lower value of $f_{\mathrm{g}} / \mathrm{B}$ for the oligomeric MMA $\left(f_{\mathrm{g}} / \mathrm{B}=0.023\right)$ is consistent with what is commonly observed in amorphous polymers of low molecular weight (Ferry 1980).

The temperature dependence of the viscosity can also be fitted using the equivalent VFT equation [equation (4)]. In this case the data are typically plotted against the quantity $1 /\left(T-T_{0}\right)$ at varied values of $T_{0}$ in order to obtain the best linear regression fit to the set of data. The data for the POSS and for the oligomer were plotted in this way and the best-fit choice of $T_{0}$ for each material is shown in Figure 9(ii). It is immediately apparent that the viscosity data over the entire temperature range conform quite well to equation (4). The calculated parameters obtained from fits to the VFT equation are shown in Table 2. The value of the Vogel temperature $T_{0}$ for the POSS $\left(T_{0}=175 \pm 9 \mathrm{~K}\right)$ is similar to that for the oligomer $\left(T_{0}=171 \pm 8 \mathrm{~K}\right)$, however the ratio $T_{0} / T_{\mathrm{g}}$, which also serves as a measure of fragility (Angell 1995), is slightly higher for the POSS. The fragility as defined by equation (3) was estimated by calculating the slope of the fits to equation (4) at $T=T_{\mathrm{g}}$. The fragilities of the POSS $(m=82)$ and the oligomer $(m=76)$ are closer in value than the fragilities obtained from DSC measurements $[m=74$ and $m=59$ respectively for the POSS and the oligomer from equation (12)]. However, the estimates 
of $m$ from the viscosity data are likely to be somewhat less accurate because the fragility parameter is defined at the glass transition temperature [equation (3)], yet all viscosity data were obtained above the glass transition, requiring an extrapolation in order to calculate $m$.

The scaling law [equation (8)] utilized by Colby (2000) was also investigated. In order to calculate the product of exponents, $v z$, the viscosity data of the pure oligomer and the pure POSS taken over the temperature range $-40^{\circ} \mathrm{C} \leq T \leq 55^{\circ} \mathrm{C}$ were plotted against the quantity $\left[\left(T-T_{\mathrm{c}}\right) / T_{\mathrm{c}}\right]$. Following the methodology described by Colby, the value of $T_{\mathrm{c}}$ was varied over a range of temperatures slightly below the glass transition temperature for each data set to obtain the best $R^{2}$ value from the linear regression fit. For the pure oligomer data, the critical temperature was determined to be $T_{\mathrm{c}}=224 \pm 5 \mathrm{~K}$, and for the POSS data $T_{\mathrm{c}}=206 \pm 7 \mathrm{~K}$. While the oligomer data are fit well by equation (8) over the entire temperature range, the POSS data are not. Only at temperatures $T<0^{\circ} \mathrm{C}$ $\left(T-T_{\mathrm{c}} \leq 65 \mathrm{~K}\right)$ do the POSS data and the fit agree. The difference between the glass transition temperature and the critical temperature are $T_{\mathrm{g}}-T_{\mathrm{c}}=2 \mathrm{~K}$ for the oligomer and $T_{\mathrm{g}}-T_{\mathrm{c}}=6 \mathrm{~K}$ for the POSS, much smaller than the magnitude of $T_{\mathrm{g}}-T_{0}$ obtained from the VFT fits from Figure 9(ii). The values for the product exponent $v z$ were found to be $10.6 \pm 1.0$ for the oligomer and $9.7 \pm 1.0$ for the methacryl-POSS. Interestingly, these values of $v z$ are very close to each other and fall within the range $8 \leq v z \leq 11$ typically observed for amorphous polymers. Colby (2000) postulated that there should be a universal dynamic scaling exponent of $v z=9$ that describes the collapse of free volume as the glass transition is approached from above and that additional "material-specific barriers to motion" could be taken into account by an Arrhenius term. In our system, in 
which the product of $v z$ is close to 9 , this Arrhenius term is small and thus the collapse of free volume appears to be the controlling factor in determining the kinetic slowdown associated with the glass transition. Overall, the dynamic scaling analysis shows that, based on the similar values of $v z$ for the methacryl-POSS and the oligomeric MMA, the glass-forming behaviors of these geometrically dissimilar molecules appear to be quite similar, which agrees qualitatively with the similar values for the fragility index $m$ calculated from fits to the VFT equation.

The data from Figure 8(i) are replotted in Figure 10 using the framework proposed by Angell that uses the ratio $T_{\mathrm{g}} / T$ to compare materials with different glass transition temperatures (Angell 1991; Angell 1995). This plot is commonly used to study the properties of glass-forming liquids in the region above $T_{\mathrm{g}}$ (Bohmer et al. 1993; Angell 1995; Angell 1997; Green et al. 1999; Debenedetti and Stillinger 2001; Ding et al. 2004). It is commonly observed that the viscosity at the glass transition is of the order $\eta_{\mathrm{g}} \sim \mathrm{O}\left(10^{12}\right) \mathrm{Pa}$ s (Angell 1995), and that the viscosity at infinite temperature asymptotes to $\eta_{\infty}=10^{-5} \mathrm{~Pa}$ s, hence our choice of limits on the ordinate scale. A strong liquid limit and a fragile liquid limit are represented on the plot. Strong liquids, such as $\mathrm{SiO}_{2}(m=$ 20), show only minor deviations from Arrhenian behavior and are characterized by tetrahedrally-coordinated structures with highly directional bonding (Angell 1995; Debenedetti and Stillinger 2001). Fragile liquids, on the other hand, generally possess isotropic bonding such as van der Waals forces and have no long-range structural order. It is apparent from Figure 11 that there is more curvature in the viscosity data of the POSS, even over the relatively small temperature range probed $\left(-55^{\circ} \mathrm{C} \leq T \leq 40^{\circ} \mathrm{C}\right)$, however the differences between the two data sets are relatively small when viewed 
within this strong-fragile framework. The fragility of the oligomer, with its short linear chain, arises from the inherently high fragility of polymers with asymmetric repeat units (Ding et al. 2004), of which PMMA is one of the most fragile with $m=145$ (Plazek et al. 1991). The fragility of PMMA is known to increase with increasing molecular weight (Ding et al. 2004), thus the lower value of $m$ for the oligomer is not surprising. The high fragility of the POSS used in this study may seem surprising in light of its significant fraction of silica content $(\approx 30 \mathrm{wt} \%)$, however the silica is present in discrete molecular cages that have no covalent connectivity. It appears that the isotropic acrylic corona is the portion of the molecule that dominates intercage interactions as the glass transition is approached.

The fact that the methacryl-POSS molecule is shaped like a cage with short arms rather than a linear chain contributes to its thermorheological properties. Materials with molecular structures of similar shape to that of the POSS (e.g. polydisperse ensembles of Lennard-Jones spheres or hyperbranched dendrimers) fall near the fragile liquid limit (Dantras et al. 2002; Murarka and Bagchi 2003). Dendrimers, for example, have been shown to exhibit enhanced enthalpy relaxation in DSC measurements (Dantras et al. 2002; see Figure 4) and also have relatively high free volumes at the glass transition temperature with $0.034 \leq f_{\mathrm{g}} / \mathrm{B} \leq 0.042$ (Dorgan et al. 2003; Tande et al. 2003). The miscible blends of oligomer and POSS exhibit an approximately constant level of $f_{\mathrm{g}} / \mathrm{B}$ at POSS loadings $\phi \leq 0.10$ before an approximately linear increase at higher loadings (Table 1). At low POSS loadings ( $\phi<0.20)$, the shape of the POSS cage is relatively unimportant because the average distance between cages is significantly greater than the diameter of the cages themselves [as we indicate schematically in Figure 11(i)]. The large 
inter-cage spacing causes the acrylic-functionalized POSS to behave like a simple plasticizer molecule. As more POSS is added, however, the cages begin to crowd each other and progressively the glass-forming properties and the viscometric properties become dominated by the geometrically-constrained POSS cages and not the oligomer chains [Figure 11(ii)]. This is particularly evident from the increase in $f_{\mathrm{g}} / \mathrm{B}$ with increasing POSS loading and the change in shape of the storage modulus curve in Figure 6(i) at $\phi_{\mathrm{POSS}}=0.20$. The POSS cages need larger void spaces through which to translate when compared with the equally large but linear oligomer chains, which can diffuse by segmental Rouse motions. The higher free volume of the POSS at $T_{\mathrm{g}}$ leads to the greater enthalpy relaxation observed in the DSC curves of Figure 4, whereby smaller $\mathrm{T}_{8} \mathrm{POSS}$ cages maintain a higher degree of mobility due to their greater ability to diffuse into the remaining pockets of free volume than the larger cages (Murarka and Bagchi 2003).

\section{Conclusion}

We have investigated the thermorheological properties of two oligomeric species of similar molecular weight and chemical affinity but different shape. One species is a linear chain [oligomeric methyl methacrylate, $M_{\mathrm{w}}=2,160 \mathrm{~g} / \mathrm{mol}$ ] and the other is an acrylic-functionalized nanocage [methacryl-POSS, $M_{\mathrm{w}}=2,025 \mathrm{~g} / \mathrm{mol}$ ]. Addition of methacryl-POSS to the MMA oligomer causes a monotonic decrease in the glass transition temperature of the blends and an increase in free volume with increasing POSS content. The DSC measurements also show that the POSS exhibits enhanced enthalpy relaxation when compared with the linear oligomer, as expected for a material with increased free volume close to $T_{\mathrm{g}}$. The enthalpy relaxation experiments were used to 
calculate the kinetic fragility index for both the POSS $(m=74)$ and the oligomeric PMMA $(m=59)$.

The viscosity of these blends over the range $T_{\mathrm{g}} \leq T \leq T_{\mathrm{g}}+100^{\circ} \mathrm{C}$ decreased monotonically with increasing POSS content. The temperature dependence of the viscosity was compared to predictions of both the free volume-based WLF-VFT framework and a dynamic scaling law in order to understand the molecular-level reasons for the observed thermorheological behavior. Fits of the available data to the WLF-VFT framework were found to be superior. Both models showed a surprising degree of similarity between the rheological behavior of the oligomeric MMA and the methacrylPOSS. The fragility of the POSS $(m=82)$ calculated from the WLF-VFT fits was only slightly larger than that of the oligomeric PMMA ( $m=76)$ and, when plotted using the framework introduced by Angell, the differences between the two species were relatively small. Overall, both species behaved as fragile liquids; of particular note was that the POSS, with its silica core, behaves nothing like a pure silica glass. This is due to its complete lack of connectivity between the silicate nanocages and the screening provided by the organic corona.

The value of $f_{\mathrm{g}}$, the fractional free volume at the glass transition temperature, determined from the reciprocal of the first WLF constant $c_{1}{ }^{\mathrm{g}}$ at the glass transition temperature, was constant at POSS loadings $\phi_{\mathrm{POSS}}<0.20$, but increased substantially at higher POSS loadings. The observed trend in $f_{\mathrm{g}}$ was rationalized by the geometric limitations imposed on neighboring POSS cages at moderate to high loadings. At low POSS loadings, the neighboring POSS cages are separated by distances greater than the cage diameter, however as the loading increases, the distance between cages decreases 
until the crowding of adjacent cages leads to additional free volume being frozen-in at the glass transition temperature.

\section{Acknowledgements}

This research was sponsored by the DURINT program of the U.S. Air Force under grant F49620-01-1-0447.

\section{References}

Adam G, Gibbs JH (1965) On the temperature dependence of cooperative relaxation properties in glass forming liquids. Journal of Chemical Physics 43:139-146.

Angell CA (1991) Relaxation in liquids, polymers, and plastic crystals - strong fragile patterns and problems. Journal of Non-crystalline Solids 131-133:13-31.

Angell CA (1995) Formation of glasses from liquids and biopolymers. Science 267:19241935.

Angell CA (1997) Why c1 = 16-17 in the WLF equation is physical - and the fragility of polymers. Polymer 38:6261-6266.

Bakac A, Brynildson ME, Espenson JH (1986) Characterization of the structure, properties, and reactivity of a cobalt(II) macrocyclic complex. Inorganic Chemistry 25:4108-4114.

Bakac A, Espenson JH (1984) Unimolecular and bimolecular homolytic reactions of organochromium and organocobalt complexes - kinetics and equilibria. Journal of the American Chemical Society 106:5197-5202.

Bird RB, Armstrong RC, Hassager O (1987) Dynamics of Polymeric Liquids, Volume 1: Fluid Mechanics, 2nd ed. New York: Wiley-Interscience.

Bohmer R, Angell CA (1992) Correlations of the nonexponentiality and state dependence of mechanical relaxations with bond connectivity in Ge-As-Se supercooled liquids. Physical Review B 45:10091-10094.

Bohmer R, Ngai KL, Angell CA, Plazek DJ (1993) Nonexponential relaxations in strong and fragile glass formers. Journal of Chemical Physics 99:4201-4208.

Capaldi FM, Rutledge GC, Boyce MC (2005) Structure and dynamics of blends of polyhedral oligomeric silsesquioxanes and polyethylene by atomistic simulation. Macromolecules 38:6700-6709.

Colby RH (2000) Dynamic scaling approach to the glass transition. Physical Review E 61:1783-1792.

Dantras E, Dandurand J, Lacabanne C, Caminade AM, Majoral JP (2002) Enthalpy relaxation in phosphorus-containing dendrimers. Macromolecules 35:2090-2094.

Debenedetti PG, Stillinger FH (2001) Supercooled liquids and the glass transition. Nature 410:259-267.

Ding Y, Novikov VN, Sokolov AP, Cailliaux A, Dalle-Ferrier C, Alba-Simionesco C, Frick B (2004) Influence of molecular weight on fast dynamics and fragility of polymers. Macromolecules 37:9264-9272. 
Dorgan JR, Knauss DM, Al-Muallem HA, Huang T, Vlassopoulos D (2003) Melt rheology of dendritically branched polystyrenes. Macromolecules 36:380-388.

Ferry JD (1980) Viscoelastic Properties of Polymers, 3rd ed. New York: John Wiley \& Sons.

Fulcher GS (1925) Analysis of recent measurements of the viscosity of glasses. Journal of the American Ceramic Society 8:339.

Green JL, Ito K, Xu K, Angell CA (1999) Fragility in liquids and polymers: new, simple quantification and interpretation. Journal of Physical Chemistry B 103:3991-3996.

Hohenberg PC, Halperin BI (1977) Theory of Dynamic Critical Phenomena. Reviews of Modern Physics 49:435.

Kelley FN, Bueche F (1961) Viscosity and glass temperature relations for polymerdiluent systems. Journal of Polymer Science 50:549.

Kopesky ET, Haddad TS, Cohen RE, McKinley GH (2004) Thermomechanical properties of poly(methyl methacrylate)s containing Tethered and Untethered Polyhedral Oligomeric Silsesquioxanes. Macromolecules 37:8992-9004.

Kopesky ET, Haddad TS, McKinley GH, Cohen RE (2005) Miscibility and Viscoelastic Properties of Acrylic Polyhedral Oligomeric Silsesquioxane-Poly(methyl methacrylate) Blends. Polymer 46:4743-4752.

Mackay ME, Dao TT, Tuteja A, Ho DL, Van Horn B, Kim HC, Hawker CJ (2003) Nanoscale effects leading to non-Einstein-like decrease in viscosity. Nature Materials 2:762-766.

Moynihan CT, Easteal AJ, DeBolt MA, Tucker J (1976) Dependence of the fictive temperature of glass on cooling rate. Journal of the American Ceramic Society 59:12-16.

Murarka RK, Bagchi B (2003) Diffusion and viscosity in a supercooled polydisperse system. Physical Review E 67:051504.

Plazek DJ, Ngai KL (1991) Correlation of polymer segmental chain dynamics with temperature-dependent time-scale shifts. Macromolecules 24:1222-1224.

Plazek DJ, Seoul C, Bero CA (1991) Diluent effects on viscoelastic behavior. Journal of Non-crystalline Solids 131-133:570-578.

Sanayei RA, O'Driscoll KF (1989) Catalytic chain-transfer in polymerization of methylmethacrylate 1. Chain-length dependence of chain-transfer coefficient. Journal of Macromolecular Science: Pure and Applied Chemistry A26:1137-1149.

Shriver DF, Drezdzon MA (1986) The Manipulation of Air Sensitive Compounds, 2nd ed. New York: Wiley-Interscience.

Stanley HE (1971) Introduction to Phase Transitions and Critical Phenomena. Oxford: Oxford University Press.

Struick LCE (1978) Physical Aging in Amorphous Polymers and Other Materials. New York: Elsevier.

Suddaby KG, Maloney DR, Haddleton DM (1997) Homopolymerizations of methyl methacrylate and styrene. Macromolecules 30:702-713.

Tammann G, Hesse W (1926) Die abhangigkeit der viskositat von der temperatur bei unterkuhlten flussigkeiten. Z. Anorg. Allg. Chem. 156:245.

Tande BM, Wagner NJ, Kim YH (2003) Influence of end groups on dendrimer rheology and conformation. Macromolecules 36:4619-4623. 
Vogel H (1921) Das temperatur-abhangigkeitsgesetz der viskositat von flussigkeiten. Phys. Zeit. 22:645.

Wang LM, Velikov V, Angell CA (2002) Direct determination of kinetic fragility indices of glassforming liquids by differential scanning calorimetry: kinetic versus thermodynamic fragilities. Journal of Chemical Physics 117:10184-10192.

Zhang Q, Archer LA (2002) Poly(ethylene oxide)/silica nanocomposites: structure and rheology. Langmuir 18:10435-10442. 
Figure 1 An image of a $T_{10}$ POSS nanocage. The methacryloxypropyl R-group is shown at the bottom left.

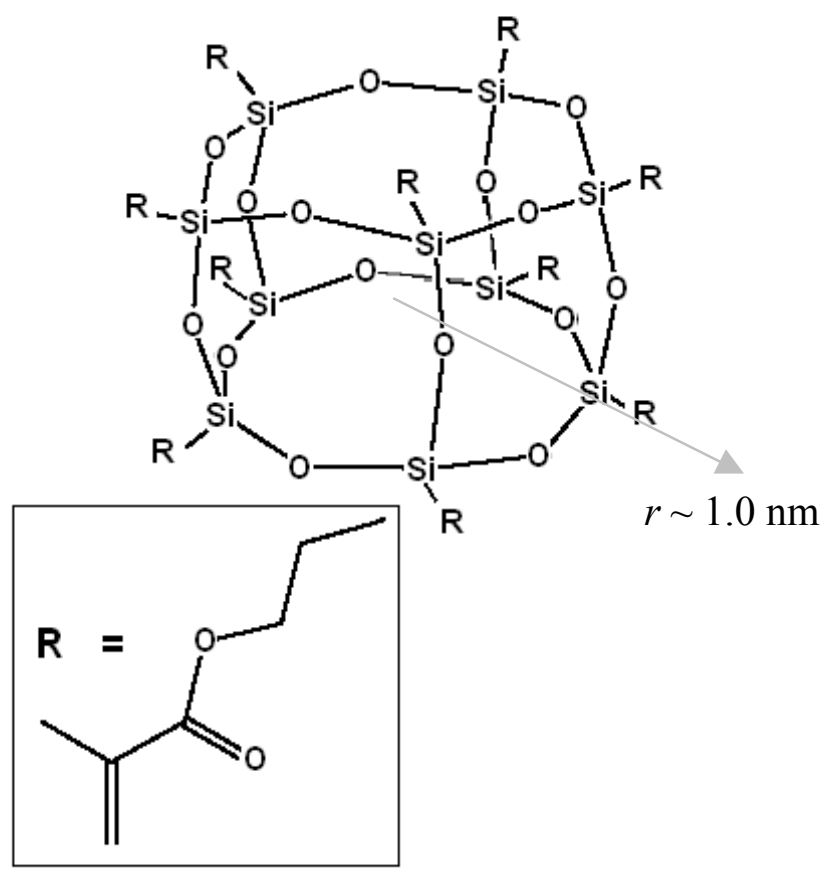


Figure 2 DSC scans for blends containing oligomeric MMA and methacryl-POSS over the composition range $0.00 \leq \phi_{\mathrm{POSS}} \leq 1.00$.

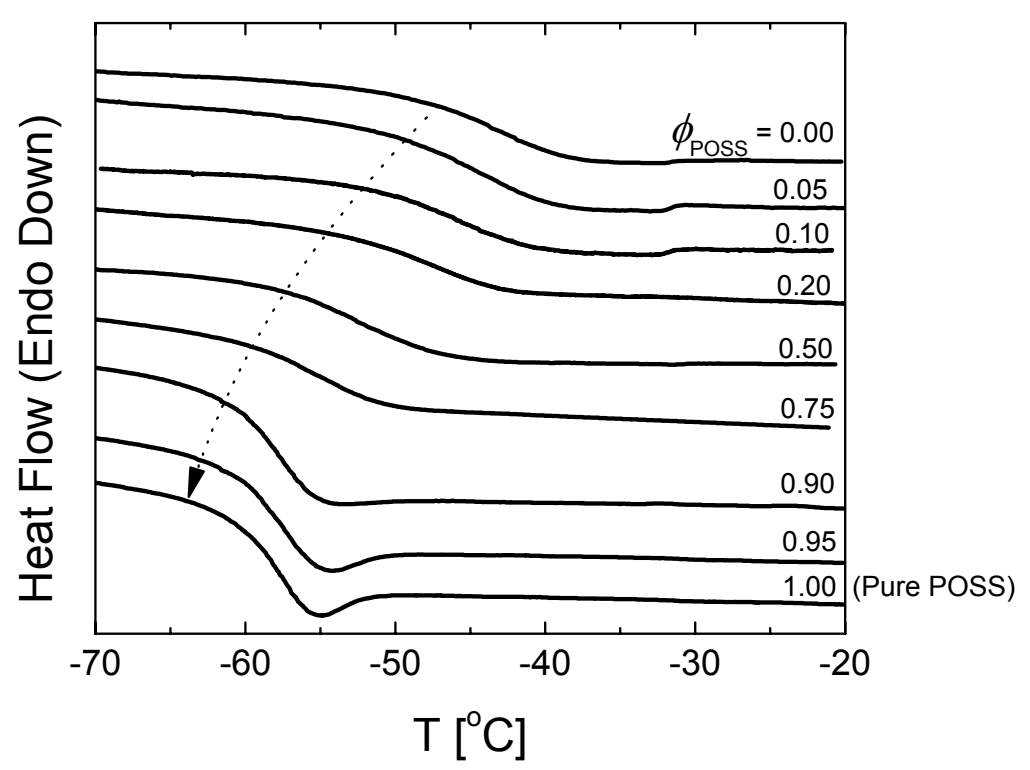


Figure 3 Values of the glass transition temperature $T_{\mathrm{g}}$ for blends containing oligomeric MMA and methacryl-POSS.

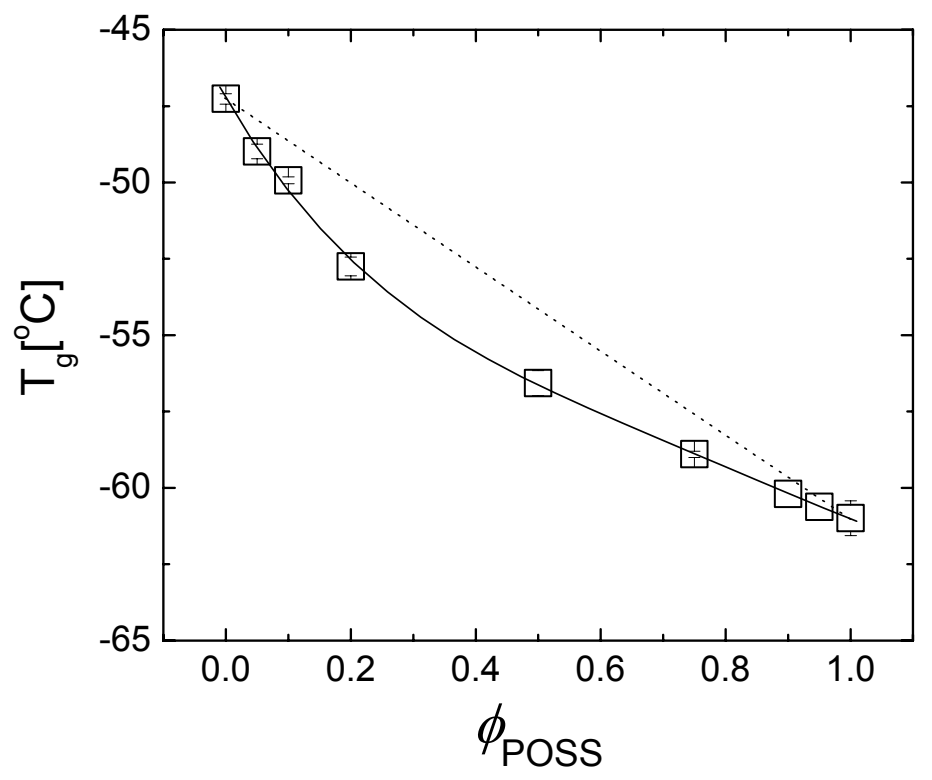


Figure 4 Specific heat capacity of the methacryl-POSS and the oligomeric MMA around the glass transition for cooling rates $q=0.2,1,4$, and $10 \mathrm{~K} \mathrm{~min}^{-1}$. The enthalpy relaxation peak decreases with increasing cooling rate. The inset shows how the limiting fictive temperature $T_{\mathrm{f}}^{\prime}$ is calculated (Moynihan et al. 1976).

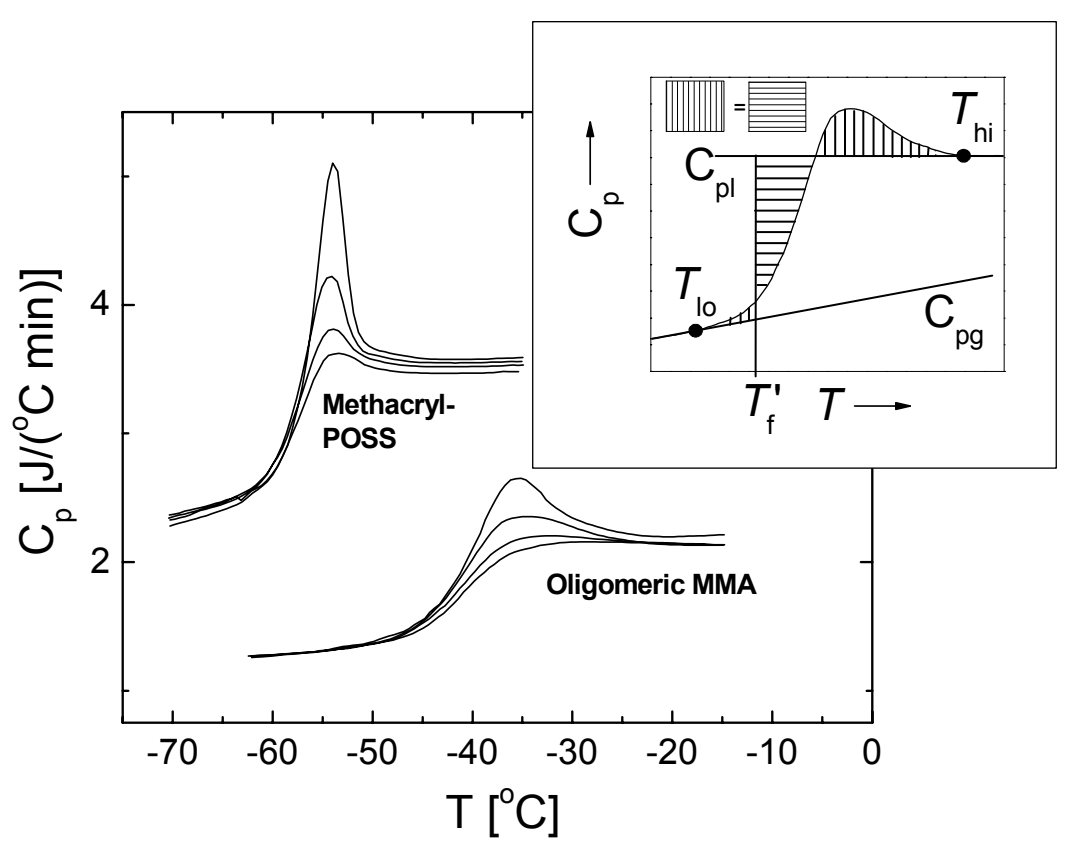


Figure 5 Natural logarithm of the cooling rate plotted against the reciprocal of the limiting fictive temperature $T_{\mathrm{f}}^{\prime}$ for oligomeric MMA and methacryl-POSS. The slope is proportional to the activation energy for structural relaxation $\Delta H^{*}$ [equation (13)]. The data for the oligomeric MMA have been shifted to the right by an additive factor of $0.001 \mathrm{~K}^{-1}$ to show more clearly the difference in slope.

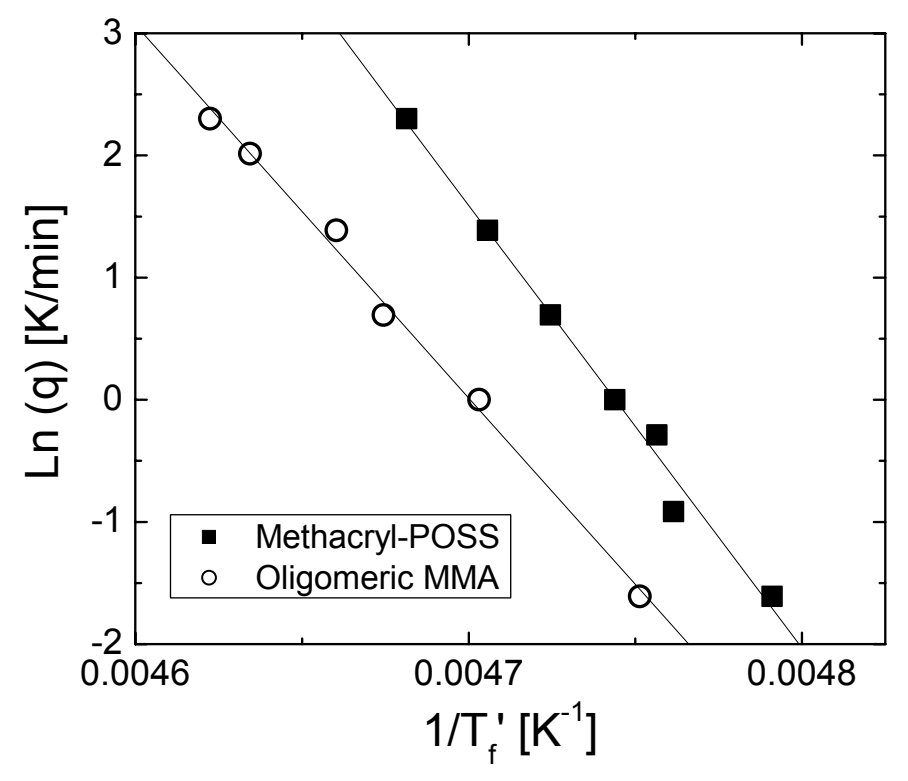


Figure 6 Values of (i) the storage moduli $G^{\prime}$ and (ii) the loss moduli $G^{\prime \prime}$ for blends of oligomeric MMA and methacryl-POSS.
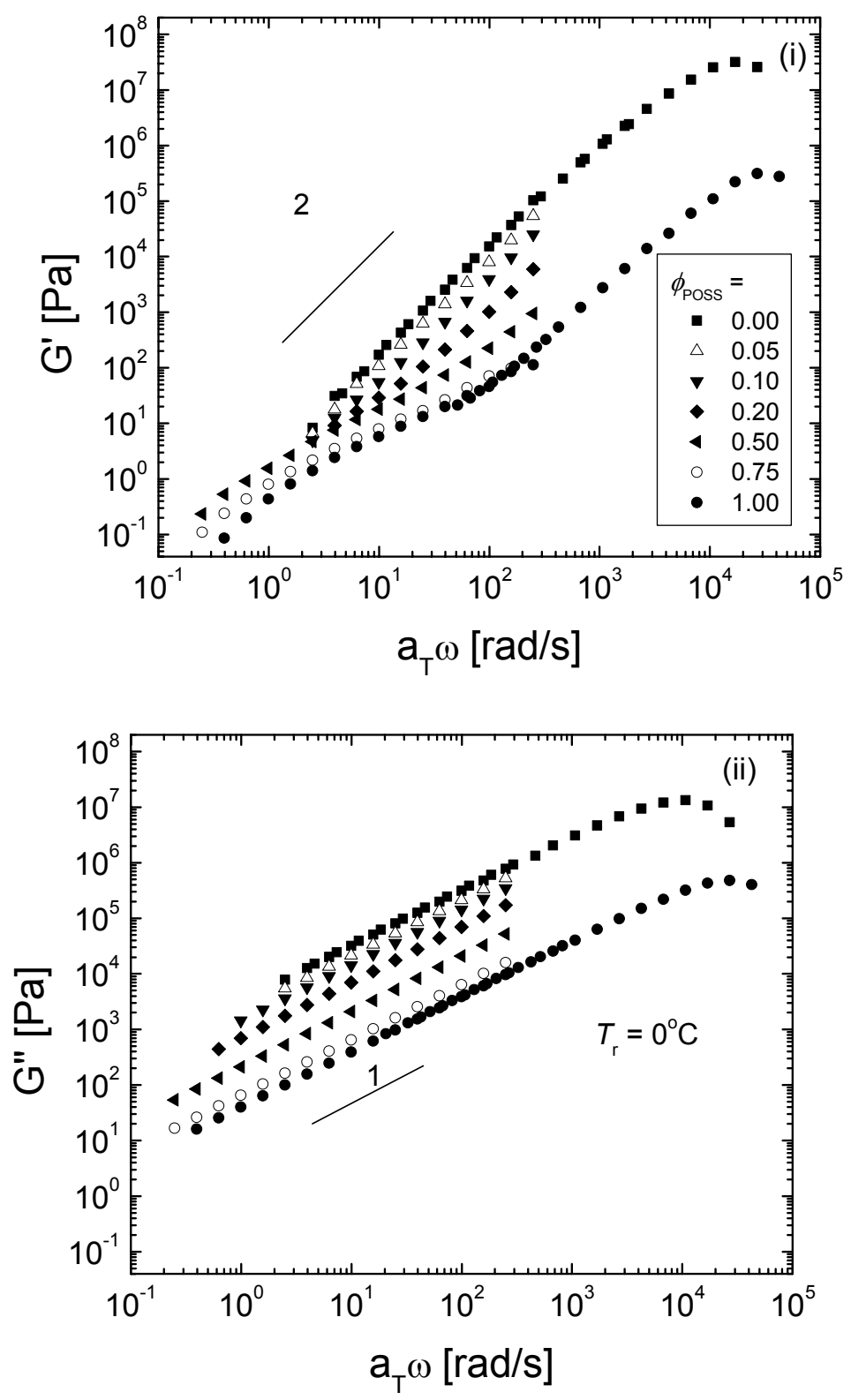
Figure 7 Zero shear-rate viscosity plotted against the volume fraction of POSS at $T=$ $20^{\circ} \mathrm{C}$.

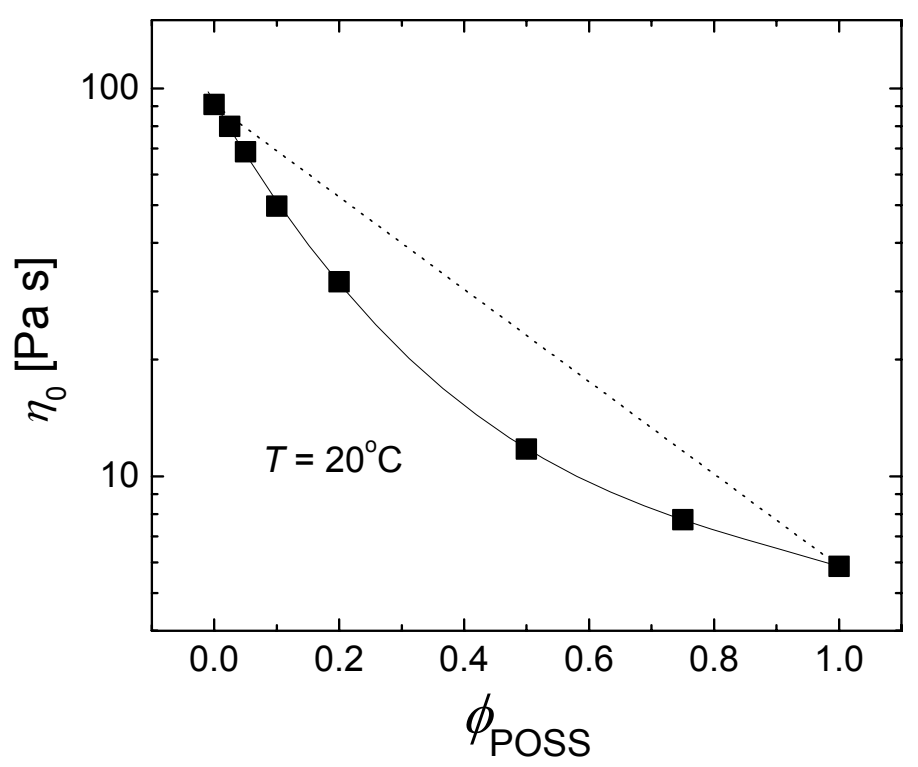


Figure 8 (i) Arrhenius plot of the zero shear-rate viscosity plotted against the reciprocal of the absolute temperature for blends of oligomeric MMA and methacryl-POSS. The dotted lines represent fits of the viscosity data for the oligomeric MMA and the methacryl-POSS over the temperature range $-40^{\circ} \mathrm{C} \leq T \leq 0^{\circ} \mathrm{C}$. (ii) Values of the flow activation energy $\Delta H$ calculated from fits to the viscosity data over the temperature range $-40^{\circ} \mathrm{C} \leq T \leq 0^{\circ} \mathrm{C}$.
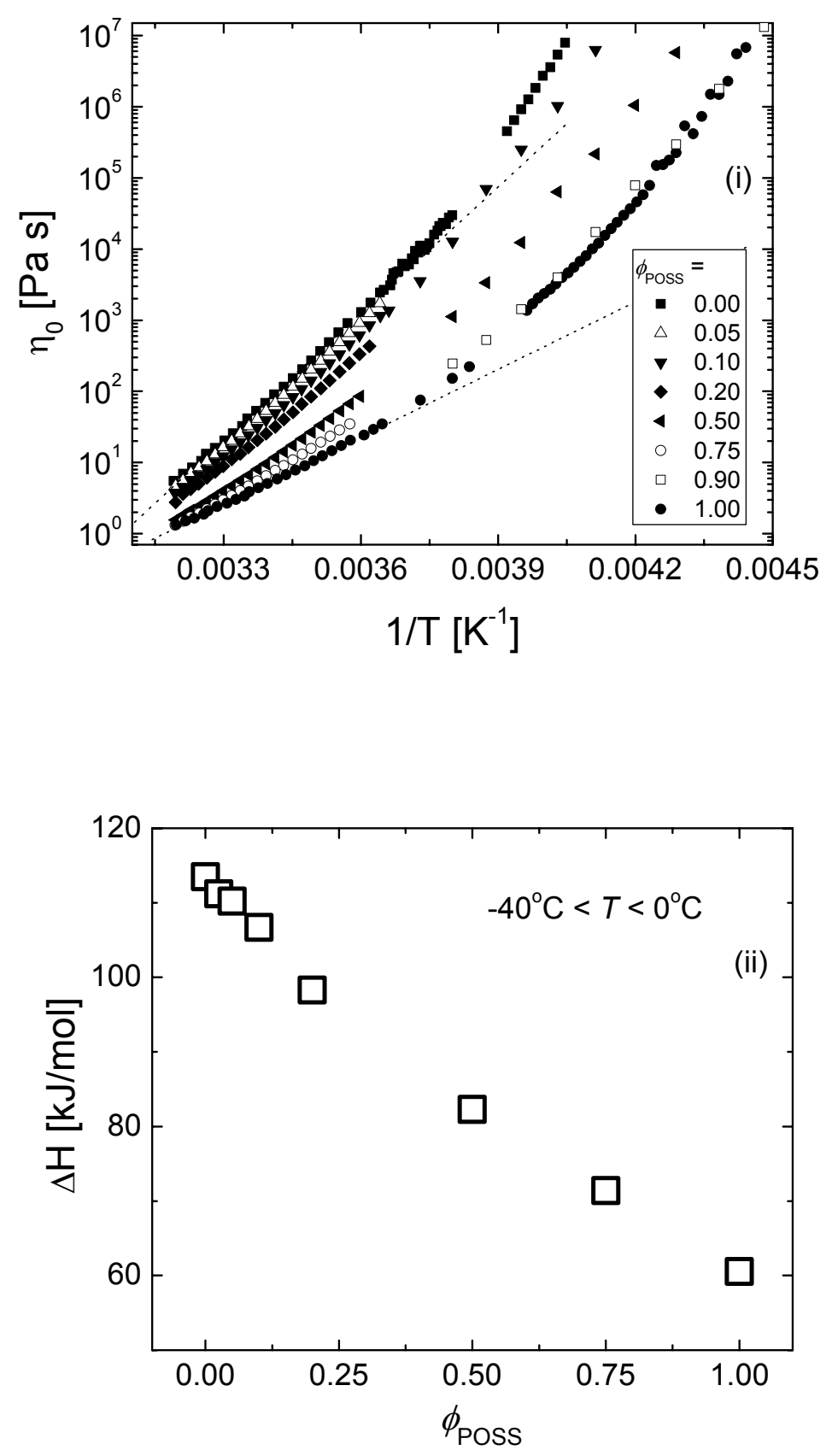
Figure 9 (i) WLF plot used to determine the constants $c_{1}$ and $c_{2}$ at a reference temperature $T_{\mathrm{r}}=20^{\circ} \mathrm{C}$. (ii) VFT plot of the zero shear-rate viscosity plotted against the quantity $1 /\left(T-T_{0}\right)$ for oligomeric MMA and methacryl-POSS.
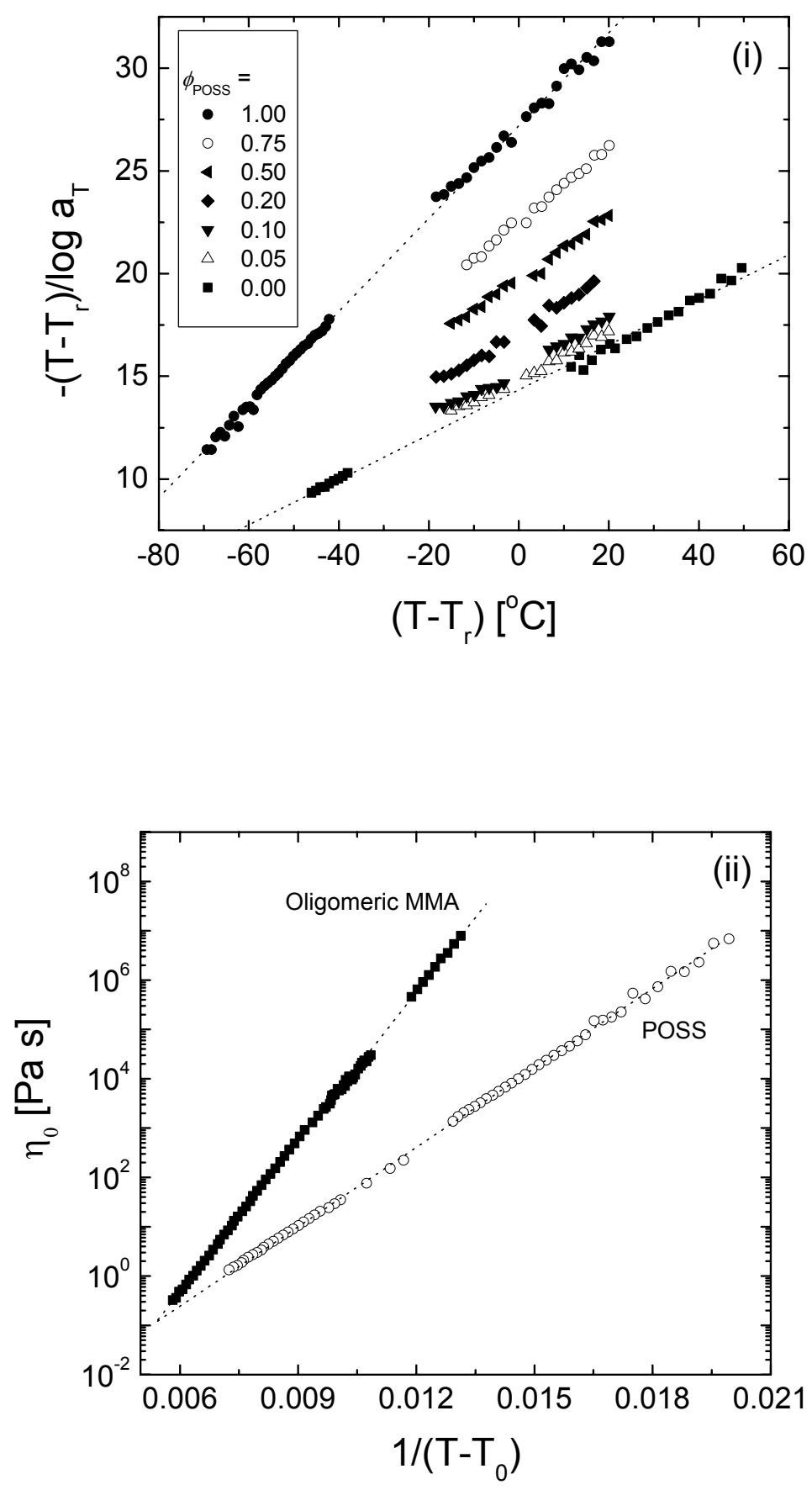
Figure 10 Angell plot of the zero shear-rate viscosity plotted against $T_{\mathrm{g}} / T$ for oligomeric MMA and methacryl-POSS. A strong liquid limit and a fragile liquid limit are also plotted.

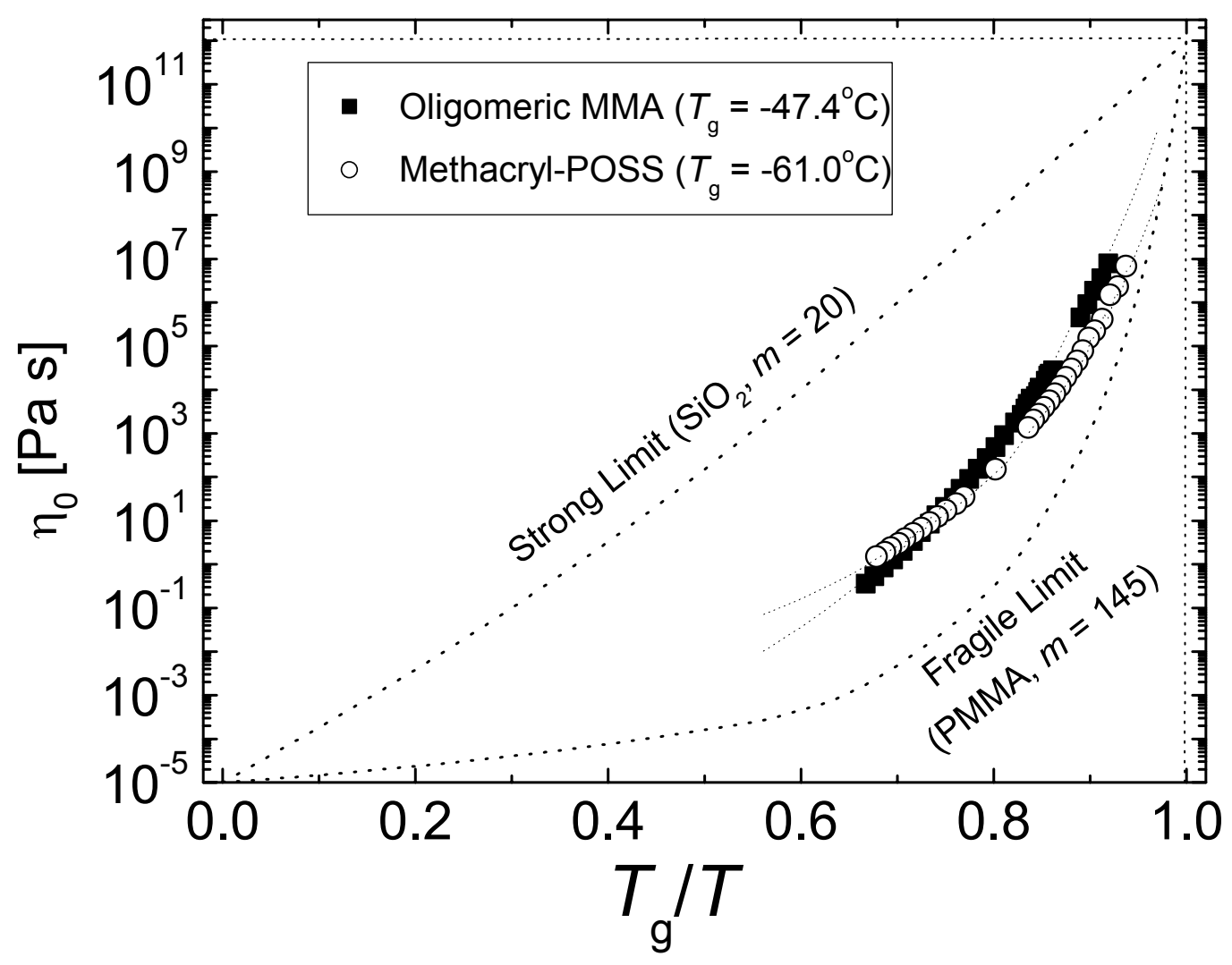


Figure 11 (i) Plasticization by POSS at low POSS loadings. At low volume fractions of $\phi_{\mathrm{POSS}}<0.20$, the distances between adjacent POSS cages are relatively large, allowing them to diffuse freely. (ii) Crowding of POSS cages at high POSS loadings, significantly hindering translational motion.

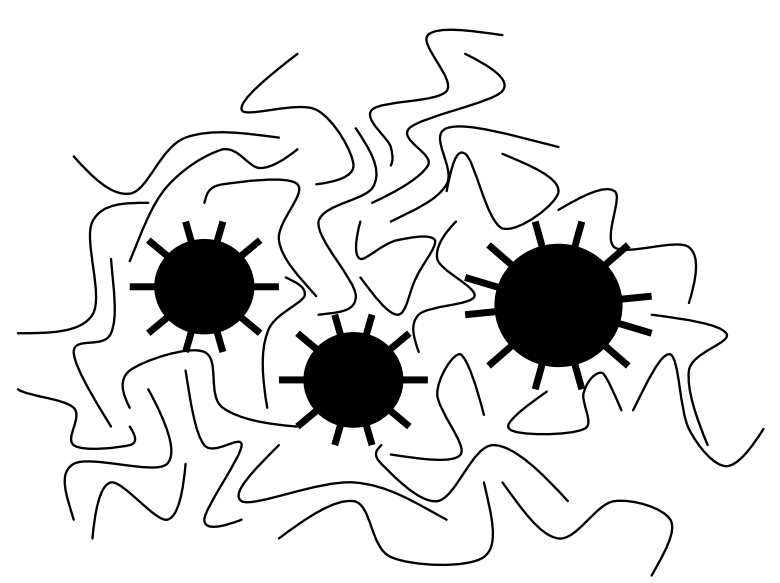

(i)

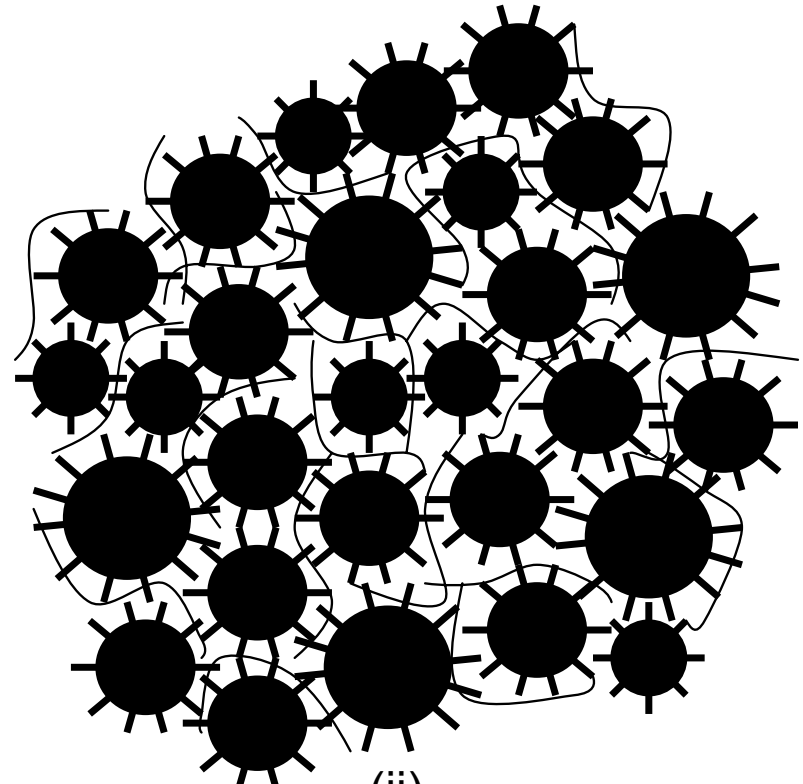

(ii) 
Tables

Table 1 - WLF Parameters for Methacryl-POSS-Oligomer Blends $\left(T_{\mathrm{r}}={ }^{\circ} 0^{\circ} \mathrm{C}\right)$

\begin{tabular}{|c|c|c|c|c|c|c|}
\hline$\phi_{\text {POSS }}$ & $c_{1}$ & $c_{2}(\mathrm{~K})$ & $T_{\mathrm{g}}\left({ }^{\circ} \mathrm{C}\right)$ & $c_{1}{ }^{9}$ & $c_{2}{ }^{9}(\mathrm{~K})$ & $f_{g} / \mathrm{B}$ \\
\hline 0.00 & 8.2 & 122.2 & -47.3 & 18.4 & 54.5 & 0.0236 \\
\hline 0.05 & 89 & 132.2 & -49.0 & 18.5 & 63.3 & 0.0234 \\
\hline 0.10 & 8.3 & 127.8 & -49.9 & 18.3 & 58.0 & 0.0237 \\
\hline 0.20 & 7.3 & 125.3 & -52.8 & 17.3 & 52.6 & 0.0250 \\
\hline 0.50 & 6.6 & 130.5 & -56.6 & 16.0 & 54.0 & 0.0272 \\
\hline 0.75 & 5.5 & 124.0 & -58.9 & 15.1 & 45.2 & 0.0287 \\
\hline 0.90 & 4.8 & 122.2 & -60.0 & 14.1 & 42.2 & 0.0309 \\
\hline 1.00 & 4.5 & 118.2 & -61.0 & 14.4 & 37.2 & 0.0302 \\
\hline
\end{tabular}

\section{Table 2 - Parameters fromfits of viscosity data} to the VFT Eqn.

\begin{tabular}{cccccc}
\hline$\phi_{\text {poss }}$ & $T_{0}(\mathrm{~K})$ & $B(\mathrm{~K})$ & $D$ & $T_{0} T_{\mathrm{g}}$ & $m$ \\
\hline 0.00 & 171 & 2320 & 13.6 & 0.76 & 76 \\
\hdashline 1.00 & 175 & 1230 & 7.0 & 0.82 & 82 \\
\hline
\end{tabular}

